\title{
Primary care professionals' satisfaction with access, communication and management advice from the diabetes team
}

Abdu Mohiddin, Smriti Naithani and Martin Gulliford Department of Public Health Sciences, Kings College, London, UK

\begin{abstract}
Most evidence on the delivery of diabetes services in primary care has focused on the organization of care rather than on the experiences of health care providers. We evaluated staff satisfaction with respect to access, communication, management advice, and understanding of roles. A postal survey of staff at 35 practices from two inner London primary care organizations was linked to profiles of the practice organization of diabetes care. Data were analysed for 124 staff including 81 doctors and 43 nurses. Only 37 (46\%) doctors and $20(47 \%)$ nurses were satisfied with the current system of diabetes care. Overall, there were low levels of satisfaction with access to, communication with, or management advice from other members of the diabetes team. Only $29 / 81(36 \%)$ of doctors understood clearly either their own role or the role of others but $27 / 43(63 \%)$ of nurses understood their own role and $23(53 \%)$ understood the role of others. Compared with practices with less satisfied staff, practices where staff were more satisfied showed similar organization of diabetes care in terms of the use of a register, recall system, clinical guidelines, availability of equipment, organization of education programmes or use of audit. Staff were generally dissatisfied with diabetes services, but the level of satisfaction was not associated with practice organization for diabetes care. Since a better team climate has been associated with higher quality care, more work should be done to understand influences on staff satisfaction in the delivery of diabetes care in primary settings.
\end{abstract}

Key words: delivery of health care; diabetes mellitus; staff satisfaction; quality of care

Received: June 2003; accepted: June 2004

\section{Introduction}

Patients with diabetes mellitus present a particular challenge to primary care as meeting the needs of patients requires the collaboration of a group of health care professionals, working together across disciplinary and organizational boundaries towards the common goal of high quality personal care (Department of Health, 1997; 2003). Randomized trials have shown that tight control of glycated haemoglobin and blood pressure are of benefit, so general practices are under increased pressure to

Address for correspondence: Dr Abdu Mohiddin, Honorary Lecturer/Consultant in Public Health Medicine, Lambeth PCT, 1 Lower Marsh, London SE1 7NT, UK.

Email: abdu.mohiddin@lambethpct.nhs.uk deliver better standards of care (Diabetes Control And Complication Trial (DCCT) Research Group, 1993; UKPDS 34, 1998). Sheldon argued that the organizational context was very important in determining the effectiveness of clinical interventions (Sheldon, 2001). Systematic review evidence has shown that diabetes care can be facilitated through organizational interventions such as computerized registers, automatic call/recall systems, dedicated clinics, or adherence to protocols, leading to improvements in processes of care, and possibly in intermediate outcomes such as lower glycatedhaemoglobin concentrations (Greenhalgh, 1994; Griffin and Kinmonth, 2000; Renders et al., 2001). A more recently developing focus in health policy has been concerned with the development of the health care workforce (Department of Health, 
2000), but this focus has not yet received as much emphasis in health care research. Evaluations of diabetes care carried out to date have generally paid little attention to the views and concerns of health care providers themselves but one study suggested that a better team climate may be associated with higher quality care (Campbell et al., 2001).

The aim of this study was to measure the levels of practice staff satisfaction with others in the multidisciplinary team with respect to: access, communication, management advice, and understanding/ clarity of roles and responsibilities; and, to describe whether satisfaction is related to practice characteristics. The study is set in the context of an evaluation of a developing shared care scheme in south London.

\section{Methods}

This study was done as part of a wider evaluation of a planned shared care scheme in two primary care groups (PCGs) in inner London. Data collection was from January 2001 to May 2002. The evaluation included questionnaire surveys of people with diabetes, practice staff, and profiles of the practices. The 220 clinical staff working in diabetes care in 54 practices in the two PCGs were sampled. As this was a substudy of the main evaluation a sample size calculation for this specific staff satisfaction element was not done. As no psychometrically robust diabetes staff satisfaction questionnaire existed at the time of the study, we developed a questionnaire for all primary care clinical staff to complete from relevant grey and published literature, and discussions with diabetes practitioners. The questionnaire asked for staff views on diabetes care including satisfaction with diabetes education and training, access to and communication with other professionals, management advice from other professionals, and understanding of their own and other professionals' roles in diabetes care.

A second questionnaire aimed to generate a profile of the practice's staffing and organization of diabetes care. This was completed either by a practice manager or a general practitioner (GP). The items included how many whole time equivalent medical and nursing staff worked at the practice; whether there was a diabetes register, a call/recall system, or a dedicated diabetes clinic; whether a range of diabetes-related staff were available on the premises, or whether there was ready referral access; availability of equipment; education for patients; and auditing of activity. Both the practice profile and staff satisfaction questionnaires were piloted with two GPs, two practice diabetes nurses, and a primary care clinical services manager. The questionnaires were posted and reminder questionnaires were sent after six weeks.

Satisfaction items were coded into a five-point Likert scale. For analysis the highest two categories were combined to denote 'satisfaction'. An overall satisfaction score was calculated by summing the 17 satisfaction items and rescaling to range from $0-100$. This satisfaction score was used to divide the practices into tertiles according to satisfaction score. Organizational characteristics of the practices were compared for the three tertiles. $P$ values were obtained using analysis of variance for continuous outcomes and Fisher's exact test for categorical variables. In the staff views questionnaire the proportion of missing values or 'don't know' responses was small (less than $3 \%$ for 14 out of 19 items). We therefore contrasted affirmative responses with negative and not known responses as described by others (Kaur et al., 1998). In the practice profile questionnaire, inspection of the pattern of responses showed that some respondents only gave affirmative responses with non-affirmative items left blank. In the analysis of this questionnaire, missing responses were therefore treated as negative.

\section{Results}

Data were analysed for 35 (65\%) practices which returned both practice profile and staff satisfaction questionnaires. The 35 practices included 185 individual staff members. Only two responses were received from professionals allied to medicine and these were excluded. There were then 124 (67\%) responses from practice staff, mean 3.5 per practice (range 1-11), including 81 doctors and 43 nurses. Responses are tabulated in Table 1. Less than half of all subjects were satisfied with the local system of diabetes care or with the education and training they had received in relation to diabetes. Considering satisfaction with access to other advice, levels of satisfaction were lowest for access to hospital diabetes specialists (39\% satisfied) and highest for access to ophthalmologists (61\%). Nurses generally expressed slightly greater satisfaction with access, 
Table 1 Primary care professionals' satisfaction diabetes care arrangements. Figures are frequencies (column percent)

\begin{tabular}{|c|c|c|c|}
\hline Satisfaction with & Doctors (81) & Nurses (43) & $P$ value \\
\hline Diabetes care system locally & $37(46)$ & $20(47)$ & 0.927 \\
\hline Diabetes education and training & $26(32)$ & $20(47)$ & 0.101 \\
\hline \multicolumn{4}{|l|}{ Access to: } \\
\hline Hospital diabetes specialist & $30(37)$ & $17(40)$ & 0.770 \\
\hline Chiropodist & $41(51)$ & $30(70)$ & 0.056 \\
\hline Dietician & $40(49)$ & $30(70)$ & 0.035 \\
\hline Diabetes specialist nurse & 40 (49) & $19(44)$ & 0.598 \\
\hline Ophthalmologist & $45(56)$ & $31(72)$ & 0.036 \\
\hline \multicolumn{4}{|l|}{ Communication with: } \\
\hline Hospital diabetes specialist & $53(65)$ & $15(35)$ & 0.003 \\
\hline Chiropodist & $30(37)$ & $15(35)$ & 0.807 \\
\hline Dietitian & $28(35)$ & $20(47)$ & 0.230 \\
\hline Diabetes specialist nurse & $33(41)$ & $15(35)$ & 0.505 \\
\hline Ophthalmologist & $47(58)$ & $23(53)$ & 0.583 \\
\hline \multicolumn{4}{|l|}{ Management advice from: } \\
\hline Hospital diabetes specialist & $52(64)$ & $19(44)$ & 0.058 \\
\hline Chiropodist & $33(41)$ & $17(40)$ & 0.897 \\
\hline Dietitian & $35(43)$ & $20(47)$ & 0.719 \\
\hline Diabetes specialist nurse & $39(48)$ & $20(47)$ & 0.859 \\
\hline Ophthalmologist & $51(63)$ & $25(58)$ & 0.548 \\
\hline Total satisfaction score & $65(58-73)$ & $65(54-76)$ & 0.601 \\
\hline $\begin{array}{l}\text { I understand clearly what my role is, and responsibilities are, } \\
\text { in providing diabetic care }\end{array}$ & $29(36)$ & $27(63)$ & 0.001 \\
\hline $\begin{array}{l}\text { I understand clearly what the roles and responsibilities are } \\
\text { of other health professionals in providing diabetic care }\end{array}$ & $29(36)$ & $23(53)$ & 0.025 \\
\hline
\end{tabular}

except for access to a diabetes specialist nurse. Satisfaction with communication with other disciplines was generally low. Doctors' expressed greatest satisfaction with communication with hospital specialists, communication with whom nurses rated poorly. Satisfaction with management advice was also low, especially for the advice received from chiropodists, dietician or diabetes specialist nurses.

The total satisfaction score was similar for medical and nursing staff. Just over one third of doctors said they had a clear understanding of their own role, or the role of others in providing diabetes care. Rather more nurses said they had a clear understanding of their own role or the role of others in diabetes care.

Table 2 provides a tabulation of the structural characteristics of general practices in relation to the satisfaction with diabetes care expressed by their staff. A majority of practices possessed a diabetes register, a system for recalling patients, guidelines for diabetes care, had referral access to specialist advice, and audited care. There was no difference in organization of care between practices with more or less satisfied staff.

\section{Discussion}

This study shows that primary care staff satisfaction was poor over a range of dimensions including access, communication, management, and education or training. We found that roles and responsibilities were not as clearly understood as desired consistent with other studies (UK Audit Commission, 2000; Campbell et al., 2001; Khunti et al., 2001) but, we noted that nurses reported more clarity with roles than doctors. The practices were generally well-organized, in terms of the possession of the characteristics associated with the delivery of effective care, but the organization of diabetes care did not vary in relation to staff satisfaction. Our results raise a question concerning the factors which influence the satisfaction of staff in delivering diabetes care.

The response rates from the practice profiles and staff views questionnaires were comparable with similar studies (Khunti and Ganguli, 2000; Pierce et al., 2000; UK Audit Commission, 2000; Campbell et al., 2001). Non-responding practices tended to be larger as in other studies (Khunti et al., 2001). 
Table 2 Structural characteristics of practices according to level of staff satisfaction. Figures are frequencies (column percent) except where indicated

\begin{tabular}{|c|c|c|c|c|}
\hline \multirow[t]{2}{*}{ Variable (missing values) } & \multicolumn{3}{|c|}{ Tertiles of satisfaction } & \multirow[t]{2}{*}{$P$-value } \\
\hline & Lowest (12) & Intermediate (12) & Highest (11) & \\
\hline Satisfaction score ${ }^{a}$ & $56(54-59)$ & $66(64-67)$ & $73(71-81)$ & - \\
\hline Number of principals ${ }^{a}(0)$ & $2(1.0-3.5)$ & $4.2(1.5-5.5)$ & $2(1-3)$ & 0.041 \\
\hline Number of nurses ${ }^{\mathrm{a}}(0)$ & $1(1-2)$ & $1.9(1.0-2.3)$ & $1(1-1.5)$ & 0.178 \\
\hline Number of items of equipment ${ }^{\mathrm{a}}(0)$ & $8(7-9)$ & $8.5(8-9)$ & $9(9-9)$ & 0.288 \\
\hline Diabetes register $(0)$ & 10 & 11 & 10 & 1.000 \\
\hline Call/recall system (8) & 7 & 7 & 7 & 1.000 \\
\hline Diabetes guidelines (7) & 9 & 8 & 8 & 1.000 \\
\hline Dedicated diabetes clinic (8) & 4 & 3 & 5 & 0.893 \\
\hline \multicolumn{5}{|l|}{ Staff on premises: } \\
\hline Named diabetes lead (16) & 2 & 5 & 4 & 0.436 \\
\hline Practice nurse (7) & 9 & 9 & 10 & 0.656 \\
\hline Diabetes specialist nurse (21) & 0 & 1 & 0 & 1.0001 \\
\hline Dietician (15) & 4 & 5 & 3 & 0.903 \\
\hline Chiropodist (16) & 2 & 6 & 1 & 0.092 \\
\hline Optometrist/optician (21) & 0 & 0 & 0 & - \\
\hline Diabetes specialist physician (21) & 0 & 1 & 0 & 1.000 \\
\hline \multicolumn{5}{|l|}{ Referral access to: } \\
\hline Diabetes specialist nurse (10) & 6 & 7 & 9 & 0.308 \\
\hline Dietician (7) & 8 & 9 & 10 & 0.470 \\
\hline Chiropodist (10) & 6 & 8 & 10 & 0.131 \\
\hline Optometrist/optician (16) & 5 & 7 & 7 & 0.589 \\
\hline Diabetes specialist physician (9) & 7 & 9 & 9 & 0.526 \\
\hline Education programmes for diabetes (2) & 4 & 4 & 4 & 1.000 \\
\hline Audit of diabetes services (4) & 9 & 8 & 7 & 0.903 \\
\hline Chronic disease management (7) & 6 & 7 & 6 & 1.000 \\
\hline
\end{tabular}

${ }^{a}$ Figures are median (interquartile range)

Non-response to individual items resulted in missing values, but a sensitivity analysis showed that alternative ways of treating missing values led to similar conclusions. The questionnaires were necessarily short. The Cronbach's alpha of the staff questionnaire is a respectable 0.88 . More in-depth qualitative work might be done to explore the views of staff in more detail and help inform the staff questionnaire's psychometric properties. The overall summed satisfaction score was used simply to separate the practices into tertiles, we feel this is justifiable as the score was comprised of complementary dimensions of 'satisfaction'. The item-by-item response can be seen in Table 1 and Table 2. Despite not doing a specific sample size calculation, some of the study findings were large enough to be detected, and may be of use in guiding and informing further research in this area.

Few studies have evaluated staff satisfaction in diabetes care. Pierce et al. (2000) found that $80 \%$ of practices said they had adequate support from their local diabetes specialist team. A more recent survey of GPs providing diabetes care by Agarwal et al. (2002) found that poor communication with secondary care, and inadequate access to specialist team members were difficulties. Our study resonates with the findings of a recent qualitative study of GPs who felt that practice organization, teamwork and access to team members were associated with quality of care (Khunti, 1999). The low level of staff satisfaction is a cause for concern for concern as good teamwork is a predictor of better quality diabetes care (Campbell et al., 2001).

This study describes a situation of low satisfaction and lack of clarity over roles. It is known that good teams are less stressed (Frith-Cozens and Payne, 1999), and further, that lower stress is known to lead to better patient care (Frith-Cozens, 2001b). Ways of improving patient care and safety, by enhancing team culture and effectiveness were outlined by Frith-Cozens et al. (2001a). These included encouraging innovation and autonomy, rewarding teams, team leadership, openness and listening, and clear management structures. As more diabetes 
care is provided under shared care schemes and by nurses, the considerably lower satisfaction of nurses (compared to doctors) when interacting with specialists (hospital and specialist nurses) suggest that this needs addressing to optimise care quality.

\section{Key points}

Primary care staff report poor satisfaction with:

- access to, communication with, and management advice from, diabetes specialists;

- their education and training in diabetes care.

There was a lack of clarity regarding the roles and responsibilities of different members of the multidisciplinary diabetes care team.

Better organization for diabetes care in a practice was not associated with greater satisfaction of staff.

\section{Acknowledgements}

This study was supported by the Charitable Foundation of Guy's and St Thomas' Hospitals. The authors thank the staff of the general practices and hospital clinics for their active participation in the study.

\section{References}

Agarwal, G., Pierce, M. and Ridout, D. 2002: The GP perspective: problems encountered in providing diabetes care in UK general practice. Diabetic Medicine 19 (Suppl. 4), 13-20.

Campbell, S.M., Hann, M., Hacker, J., Burns, C., Oliver, D., Thapar, A., Mead, N., Safran, D.G. and Roland, M.O. 2001: Identifying predictors of high quality care in English general practice: observational study, British Medical Journal 323, 784-87.

Department of Health. 1997: Health Service Guidelines, Key Features of a good service. HSG (97): Department of Health.

Department of Health. 2000: A health service of all the talents developing the NHS workforce consultation document on the review of workforce planning. Leeds, UK: Department of Health.
Department of Health. 2003: National service framework for diabetes: delivery strategy, Department of Health.

Diabetes Control And Complication Trial (DCCT) Research Group. 1993: The effect of intensive treatment of diabetes on the development and progression of long term complications in insulin dependent diabetes mellitus. New England Journal of Medicine 329, 977-86.

Frith-Cozens, J. 2001a: Cultures for improving patient safety through learning: the role of teamwork. Quality in Health Care 10 (Suppl. 1), ii26-ii31.

Frith-Cozens, J. 2001b: Interventions to improve physicians' wellbeing and patient care. Social Science and Medicine 52, 215-22.

Frith-Cozens, J. and Payne, R.L. 1999: Sharing the burden: team work in health care settings. In Frith-Cozen, J. and Payne, R.L. editors. Stress in health professionals: psychological and organizational causes and interventions. John Wiley.

Greenhalgh, T. 1994: Shared Care for Diabetes. Occ Paper 67. The Royal College of General Practitioners.

Griffin, S. and Kinmonth, A.L. 2000: Diabetes care: the effectiveness of systems for routine surveillance for people with diabetes. [Review] [5 refs]. Cochrane database of systematic reviews [Computer File] 2, CD000541.

Kaur, B., Anderson, H.R., Austin, J., Burr, M., Harkins, L.S. and Strachan, D.P. 1998. Prevalence of asthma symptoms, diagnosis, and treatment in 12-14 year old children across Great Britain (International Study of Asthma and Allergies in Childhood, ISAAC, UK). British Medical Journal 316, 118-24.

Khunti, K. 1999: Use of multiple methods for assessing quality of care. Family Practice 16, 489-94.

Khunti, K. and Ganguli, S. 2000: Who looks after people with diabetes: primary or secondary care? Journal of the Royal Society of Medicine 93, 183-86.

Khunti, K., Ganguli, S., Baker, R. and Lowy, A. 2001: Features of primary care associated with variations in process and outcome of care of people with diabetes. British Journal of General Practice 51, 356-60.

Pierce, M., Agarwal, G. and Ridout, D. 2000: A survey of diabetes care in general practice in England and Wales. British Journal of General Practice 50, 542-45.

Renders, C.M., Valk, G.D., Griffin, S., Wagner, E.H., Eijk, J. and Assendelft, W.J.J. 2001: Interventions to improve the management of diabetes mellitus in primary care, outpatient and community settings. The Cochrane Library.

Sheldon, T. 2001: It ain't what you do but the way that you do it. Journal of Health Service and Research policy 6, 3-5.

UK Audit Commission. 2000: Testing times: a review of diabetes services in England. London: UK Audit Commission.

UKPDS 34. 1998: UK Prospective Diabetes Study Group. Intensive blood glucose control with sulphonyureas or insulin compared with conventional therapy and risk of complications in patients with type 2 diabetes. Lancet 352, 854-65. 